\title{
New Stishovite-like Phase of Silica formed by Hydrostatic Compression of Cristobalite
}

\author{
By Masaaki YAMAKATA*) and Takehiko YAGI \\ Institute for Solid State Physics, University of Tokyo, Minato-ku, Tokyo 106 \\ (Communicated by Syun-iti Aкıмото, M. J. A., June 12, 1997)
}

\begin{abstract}
In order to clarify the effect of non-hydrostaticity of pressure on the crystal lattice of minerals, various pressures with different hydrostaticity were applied to silica (cristobalite) at room temperature and high pressure in situ $\mathrm{x}$-ray observations were made. Under strongly non-hydrostatic compression, cristobalite transformed to an "X-I phase" reported in the previous study, while under quasi-hydrostatic compression, a new polymorph was synthesized. Although the new polymorph gives very similar diffraction profile to stishovite, its unit cell dimension is meaningfully different from that of stishovite. Present study clarified that in cristobalite the crystal structure of high pressure polymorph changes completely depending on the hydrostaticity of applied pressure.
\end{abstract}

Key words: Silica; hydrostaticity; high pressure; in situ x-ray observation.

Introduction. All materials solidify at pressures above $10 \mathrm{GPa}$ at room temperature. ${ }^{1)}$ High pressure experiments above this pressure have been carried out under non-hydrostatic condition, though there is wide variety of non-hydrostaticity depending on the method of high pressure generation. The variety of non-hydrostaticity may cause the differences on compressibility of materials or the transition pressure to high pressure phase. In fact, it has been observed that the compressibility of stishovite ${ }^{2)-5)}$ and the transition pressure from stishovite to $\mathrm{CaCl}_{2}$-type phase ${ }^{4), 6)}$ differ considerably depending on the experiments, and it leads to the confusion in comparing the experimental results. Use of synchrotron radiation has made it possible to carry out the accurate $\mathrm{x}$ ray diffraction study under high pressure, and thus very small distortions of crystal lattice due to the nonhydrostaticity can be now observed. ${ }^{7), 8}$ In high pressure experiments, it is now important not only to generate very high pressure but also to control the hydrostaticity of pressure. In the present study, the behavior of silica $\left(\mathrm{SiO}_{2}\right)$ was clarified through high pressure in situ $\mathrm{x}$-ray diffraction under various degree of non-hydrostaticity to clear the effect of non-hydrostaticity on the crystal lattice of mineral.

Silicate minerals are believed to be the main components of the Earth's mantle, and silica $\left(\mathrm{SiO}_{2}\right)$ is one

*) Present address: Japan Atomic Energy Research Institute, SPring-8, Kamigori-cho, Ako-gun, Hyogo 678-12, Japan. of the most basic silicate minerals. In spite of its simple chemical formula, there are many polymorphs in silica. Cristobalite is one of the high temperature polymorphs of silica and has a very loose structure. The behavior of cristobalite under pressure has been studied by a computer simulation and a compression experiment. These studies, however, showed interesting contrast. The molecular dynamic calculation showed that cristobalite transforms into stishovite at about $20 \mathrm{GPa}$ directly or via an intermediate phase with a space group of $\mathrm{Cmcm} .{ }^{9)}$ On the other hand, high pressure experiment clarified that cristobalite transforms into unknown structured phase named "X-I" when the sample was compressed at room temperature by diamond anvil cell without using any pressure transmitting medium. ${ }^{10)}$ The reason of this discrepancy is not yet clear, but one possible explanation is that the pressure achieved in the diamond anvil cell was highly non-hydrostatic uniaxial stress, while that simulated in computer was hydrostatic. In order to check this possibility, the room temperature compression of cristobalite was carried out by changing the hydrostaticity of applied pressure.

Experimental. All the experiments were made using modified Mao-Bell type diamond anvil cell. ${ }^{11)}$ Rhenium gasket was compressed to a thickness of $60 \mu \mathrm{m}$ using diamond anvils with a culet size of $0.45 \mathrm{~mm}$. A hole with $200 \mu \mathrm{m}$ in diameter was made in the center of the indentation of gasket and used as a sample chamber. 
Pressure was measured using ruby fluorescence method. ${ }^{12)}$ In diamond anvil cell, it is possible to use gaseous material, such as $\mathrm{Ar}$ and $\mathrm{Ne}$ as a pressure transmitting medium, and they give quasi-hydrostatic environment even above their solidifying pressures. ${ }^{13)}$ It is thought that the hydrostaticity of pressure was controlled by changing the relative quantity of pressure transmitting medium to the sample. In the present experiment with high hydrostaticity, sample chamber was filled with a large amount of gaseous material such as $\mathrm{Ar}, \mathrm{Ne}$, or $\mathrm{H}_{2} \cdot{ }^{14)} \mathrm{On}$ the other hand, for the experiment under highly nonhydrostatic condition, powdered sample was directly compressed without using any pressure transmitting medium. In order to achieve an intermediate hydrostatic condition between quasi-hydrostatic and uniaxial compression, the sample chamber was filled with a small amount of Ar. Hydrostaticity of pressure was checked by the pressure distribution in the sample chamber and the line width of ruby fluorescence line. ${ }^{1)}$ In fact, when the sample chamber was filled with large amount of pressure transmitting medium, there was no observable pressure distribution and the line width of the ruby fluorescence remained unchanged. The line width of ruby fluorescence increased with decreasing the amount of the pressure transmitting medium. In the compression without pressure transmitting medium, the pressure difference from the center to the edge of the sample chamber was as large as $10 \%$. From these observations, it is clear that the hydrostaticity of pressure varies with the relative amount of the pressure transmitting medium and the sample.

Cristobalite was prepared by firing precipitated $\mathrm{SiO}_{2}$ at $1550^{\circ} \mathrm{C}$ for 5 hours and then quenching it in liquid nitrogen. All the high pressure in situ $\mathrm{x}$-ray observations were made using synchrotron radiation at the Photon Factory, KEK. A monochromatized $x$-ray $(\lambda=0.6888 \AA$ ) was collimated to a thin beam of $100 \mu \mathrm{m}$ in diameter and was irradiated to the sample. Diffracted $x$-ray was recorded on an imaging plate detector ${ }^{15)}$ placed $150 \mathrm{~mm}$ apart from the sample. The detail of the $\mathrm{x}$-ray experiment was described elsewhere. ${ }^{16)}$

Results and discussion. Under quasi-hydrostatic compression, cristobalite reduced its volume monotonously with increasing pressure and preserved its crystal structure until the pressure reached $18 \mathrm{GPa}$. When the pressure was increased above $18 \mathrm{GPa}$, new diffraction lines appeared, which are similar to those of rutile-type structure phase (stishovite). As will be discussed later in detail, the unit cell dimensions are meaningfully different with those of normal stishovite formed under high temperature and pressure. Moreover, there always exists

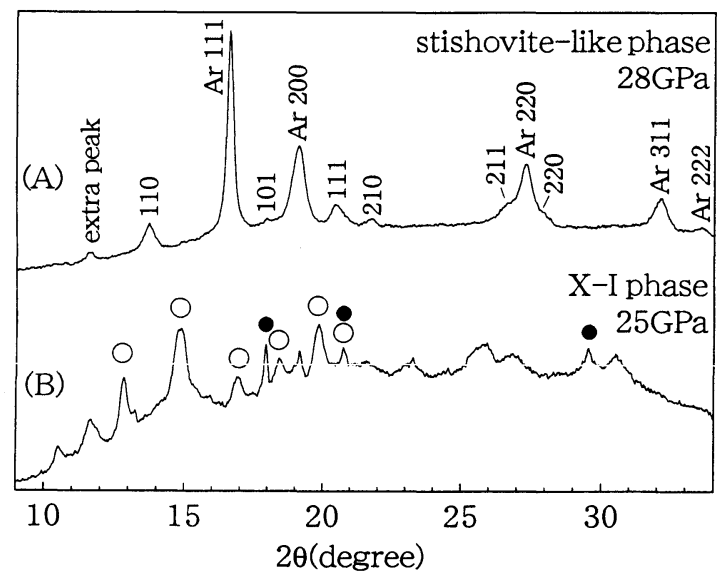

Fig. 1. Examples of the x-ray diffraction profiles obtained by (A) quasi-hydrostatic compression at about $28 \mathrm{GPa}$ and (B) uniaxial compression at about $25 \mathrm{GPa}$. In (A), diffractions from Ar pressure transmitting medium and an extra peak, which cannot be indexed by rutile-type structure, are observed. In (B), diffractions reported in the previous study ${ }^{10)}$ and those from the platinum powder mixed with the specimen are shown by open and solid circles, respectively.

an extra diffraction line $(\mathrm{d} \sim 3.5 \AA)$ which cannot be indexed by the rutile-type structure (Fig. 1(A)). This "stishovitelike" phase was preserved even after the pressure was completely released and its calculated unit cell parameters based on the indexing of rutile-type structure is shown in Table I(A). No difference of the result was found in the experiments using either $\mathrm{Ar}, \mathrm{Ne}$, or $\mathrm{H}_{2}$ as pressure transmitting medium.

When the sample was compressed without any pressure transmitting medium, cristobalite transformed into different high pressure phase called "X-I" above 10 $\mathrm{GPa}$, in accordance with the previous study. ${ }^{10)}$ Observed diffraction lines (Fig. 1(B)) are almost the same as those reported before, though some additional weak diffraction lines were observed in the present study, which is a result of higher resolution and high sensitivity of the present $\mathrm{x}$ ray diffraction system. Because of the broad nature of the diffraction lines, however, it is still difficult to determine the crystal structure based on this diffraction profile.

When the sample was compressed with a small amount of pressure transmitting medium, both X-I phase and untransformed cristobalite were observed at about 15 $\mathrm{GPa}$. This mixture transformed into X-I phase plus "stishovite-like" phase at about $20 \mathrm{GPa}$. It is clear that the high pressure phases formed under quasi- and nonhydrostatic environments coexist under this intermediate condition.

In Fig. 2, pressure dependence of the unit cell parameters of "stishovite-like" phase are compared with 
Table I. An x-ray diffraction data of "stishovite-like" phase indexed according to (A) rutile-type structure and (B) super lattice structure of rutile-type structure

\begin{tabular}{|c|c|c|c|c|c|c|c|c|c|c|}
\hline \multicolumn{11}{|c|}{ X-ray diffraction data } \\
\hline \multirow{3}{*}{$\begin{array}{l}\mathrm{d}_{\mathrm{obs}}(\AA \AA) \\
3.539\end{array}$} & \multicolumn{5}{|c|}{ (A) Rutile-type structure $(Z=2)$} & \multicolumn{5}{|c|}{ (B) Super lattice structure $(Z=18)$} \\
\hline & $\mathrm{h}$ & $\mathrm{k}$ & 1 & $\mathrm{~d}_{\mathrm{cal}}(\AA)$ & $\mathrm{d}_{\mathrm{obs}} / \mathrm{d}_{\mathrm{cal}}-1$ & $\mathrm{~h}$ & $\mathrm{k}$ & 1 & $\mathrm{~d}_{\mathrm{cal}}(\AA \AA)$ & $\mathrm{d}_{\mathrm{obs}} / \mathrm{d}_{\mathrm{cal}}-1$ \\
\hline & & & & -- & & 3 & 2 & 0 & 3.519 & 0.006 \\
\hline 2.981 & 1 & 1 & 0 & 2.983 & -0.001 & 3 & 3 & 0 & 2.991 & -0.003 \\
\hline 2.251 & 1 & 0 & 1 & 2.250 & 0.001 & 3 & 0 & 1 & 2.250 & 0.001 \\
\hline 1.985 & 1 & 1 & 1 & 1.985 & 0.000 & 3 & 3 & 1 & 1.985 & 0.000 \\
\hline 1.537 & 2 & 1 & 1 & 1.539 & -0.001 & 6 & 3 & 1 & 1.541 & -0.003 \\
\hline \multirow[t]{2}{*}{1.494} & 2 & 2 & 0 & 1.492 & 0.001 & 6 & 6 & 0 & 1.495 & -0.001 \\
\hline & \multicolumn{5}{|c|}{$\begin{array}{l}\text { Calculated unit cell } \\
\mathrm{a}=4.219(4)(\AA) \\
\mathrm{c}=2.660(4)(\AA) \\
\mathrm{v}=47.3(1)\left(\AA^{3}\right)\end{array}$} & \multicolumn{5}{|c|}{$\begin{array}{l}\text { Calculated unit cell } \\
\mathrm{a}=12.69(3)(\AA) \\
\mathrm{c}=2.655(1)(\AA) \\
\mathrm{v}=427(2)\left(\AA^{3}\right)\end{array}$} \\
\hline
\end{tabular}

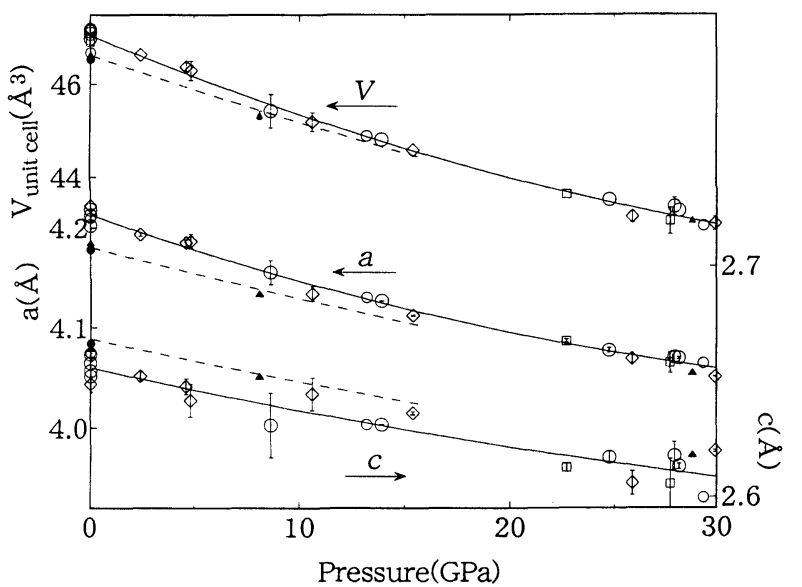

Fig. 2. Pressure dependences of the unit cell parameters of "stishovite-like" phase formed using various pressure transmitting media (open symbols; $\bigcirc \mathrm{Ar}, \diamond \mathrm{Ne}, \square \mathrm{H}_{2}$ ) and stishovite formed by heating (solid symbols; $\boldsymbol{\Delta} 300^{\circ} \mathrm{C}$, $1000^{\circ} \mathrm{C}$ ). Solid line and dashed line represent compression curves of "stishovite-like" phase and stishovite observed by Ross et al., "5) respectively.

that of normal stishovite. The results are scattered due to the broad nature of the observed diffraction lines but it is clear that the unit cell volume of "stishovite-like" phase at ambient condition is about $1.2 \%$ larger compared to that of normal stishovite. ${ }^{5)}$ Moreover, $a$-axis and $c$-axis are, respectively, slightly elongated and compressed, resulting smaller c/a ratio of tetragonal unit cell. It is known that when iron was compressed in hydrogen, the unit cell volume of iron increased considerably due to the dissolution of hydrogen into iron lattice. ${ }^{17)}$ In order to check similar possibility, the experiment was repeated using different kind of pressure transmitting medium. As mentioned before, identical results were obtained using either $\mathrm{Ar}, \mathrm{Ne}$, or $\mathrm{H}_{2}$ as pressure transmitting medium. This indicates that it is difficult to explain the difference of the unit cell dimensions by the dissolution or chemical reaction with these materials. Chemical analysis of the recovered sample using EPMA also indicates no sign of chemical contamination. It can be concluded that the difference of these unit cell dimesions are caused by the difference of crystal structure. In previous studies, it is sometimes reported that a distortion from tetragonal symmetry to monoclinic one was observed between 1 to 2 $\mathrm{GPa}$ during the room temperature compression of cristobalite, by means of Raman, IR, ${ }^{18), 19)}$ and x-ray diffraction studies. ${ }^{20)-22)}$ No clear evidence of such distortion was observed in the present study, in accordance with our previous study. ${ }^{10)}$ The reason of this difference is not yet clear. It could be explained either by the difference of starting material or by the difference of experimental method.

When the "stishovite-like" phase was observed, an extra diffraction line, other than those indexed by rutiletype structure, was always observed at about $3.5 \AA$ of $d-$ spacing. It was impossible to explain the diffraction line by other polymorphs of silica based on the d-spacing and its compressibility. The compressibility of this line was similar to that of $a$-axis of rutile-type unit cell. This suggests that the "stishovite-like" phase could be explained by a super lattice structure of stishovite. Possibility to index this line was searched by multiplying the unit cell dimension along the $a$-axis of rutile-type unit cell. The diffraction line can be reasonably explained by assuming larger unit cell in which $a$-axis is elongated three times than the original, though the agreement of observed and calculated d-spacings are 


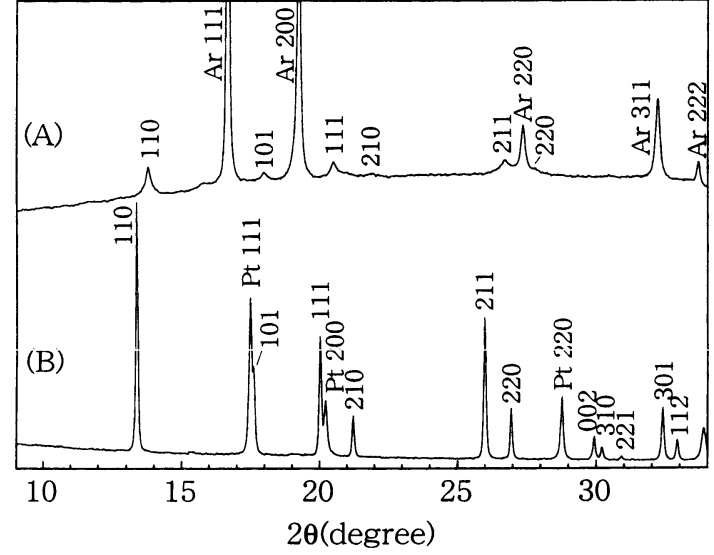

Fig. 3. Example of the x-ray diffraction profiles of stishovite formed by heating under pressure of $30 \mathrm{GPa}$. These profiles are observed at (A) $29 \mathrm{GPa}$ after heating to $300^{\circ} \mathrm{C}$ and (B) atmospheric pressure after heating to $1000^{\circ} \mathrm{C}$ under pressure, respectively.

still not so good (Table I(B)). Electron diffraction pattern of this phase was also studied using transmission electron microscope but it was still difficult to get clear diffraction to make any conclusion of the super lattice structure, due to the poor crystallinity. Very broad nature and low $\mathrm{S} / \mathrm{N}$ ratio of the x-ray diffraction also made it difficult to make further discussion of the crystal structure.

When this "stishovite-like" phase was heated approximately $300^{\circ} \mathrm{C}$ under high pressure of $30 \mathrm{GPa}$, the extra diffraction line $(\mathrm{d} \sim 3.5 \AA)$ disappeared and all other lines became slightly sharp, as shown in Fig. 3(A). Much sharper diffraction lines were obtained when it was heated above $1000^{\circ} \mathrm{C}$ using YAG laser, which is a common technique to synthesize normal stishovite (Fig. 3(B)). The diffraction profiles after heating are well indexed by the rutile-type structure and its unit cell dimensions are in good agreement with stishovite (Fig. 2). These observations strongly indicate that the "stishovite-like" phase formed by the room temperature quasi-hydrostatic compression is a metastable phase formed by the luck of kinetic energy.

In the present study, it became clear that the crystal structure of high pressure polymorph sometimes changes completely depending on the hydrostaticity of applied pressure. This means that careful consideration of the nature of applied pressure is required to study the stable crystal structure under pressure. Present result also indicates that the result of molecular dynamic calculation is in better agreement with the experimental result obtained under quasi-hydrostatic condition. It may be quite interesting to make molecular dynamic calculation which simulate the compression under non-hydrostatic condition.

Acknowledgments. We thank Drs. T. Kondo, S. Tsuneyuki, and W. Utsumi for helpful comments and discussions throughout this research. Thanks are also due to Prof. S. Akimoto, M. J. A., for encouragement and helpful comments to this study. X-ray experiments were carried out at KEK (95G110). M. Yamakata has been supported by research Fellowships of the Japan Society for the Promotion of Science for Young Scientist.

\section{References}

1) Piermarini, G. J., Block, S., and Barnett, J. D. (1973) J. Appl. Phys. 44, 5377-5382.

2) Liu, L. G., Bassett, W. A., and Takahashi, T. (1974) J. Geophys. Res. 79, 1160-1164.

3) Sato, Y. (1977) Earth Planet. Sci. Lett. 34, 307-312.

4) Tsuchida, Y., and Yagi, T. (1989) Nature 340, 217-220.

5) Ross, N. L., Shu, J. F., and Hazen, R. M. (1990) Am. Mineral. 75, 739-747.

6) Mao, H. K., and Hemley, R. J. (1996) Phil. Trans. R. Soc. London A354, 1315-1332.

7) Funamori, N., Yagi, T., and Uchida, T. (1994) J. Appl. Phys. 75, 4327-4331.

8) Uchida, T., Funamori, N., and Yagi, T. (1996) J. Appl. Phys. 80, 739-746.

9) Tsuneyuki, S., Matsui, Y., Aoki, H., and Tsukada, M. (1989) Nature 339, 209-211.

10) Tsuchida, Y., and Yagi, T. (1990) Nature 347, 267-269.

11) Yagi, T., and Akimoto, S. (1982) In High-Pressure Research in Geophysics (eds. Akimoto, S., and Manghnani, M.). CAPJ, Tokyo, pp. 81-90.

12) Mao, H. K., Bell, P. M., Shaner, J. W., and Steinberg, D. J. (1978) J. Appl. Phys. 49, 3276-3283.

13) Bell, P. M., and Mao, H. K. (1981) Year book Carnegie Inst. Washington 80, 404-406.

14) Yagi, T., Yusa, H., and Yamakata, M. (1996) Rev. Sci. Instrum 67, 2981-2984.

15) Miyahara, J., Takahashi, K., Amemiya, Y., Kamiya, Y., and Satow, Y. (1986) Nucl. Instr. Methods A246, 572-578.

16) Yagi, T., Uchiyama, Y., Akaogi, M., and Ito, E. (1992) Phys. Earth Planet Inter. 74, 1-7.

17) Yamakata, M., Yagi, T., Utumi, W., and Fukai, Y. (1992) Proc. Japan Acad. 68B, 172-176.

18) Sugiura, H., and Yamadaya, T. (1989) Prog. and Abstrs. 30th High Pressure Symp., pp. 162-163.

19) Yahagi, Y., Yagi, T., Yamawaki, H., and Aoki, K. (1994) Solid State Commun. 89, 945-948.

20) Parise, D. J., Yaganeh-Haeri, J. A., and Weidner, D. J. (1994) J. Appl. Phys. 75, 1361-1367.

21) Palmer, D. C., and Finger, L. W. (1994) Am. Mineral. 79, 1-8.

22) Downs, R. T., and Palmer, D. C.(1994) Am. Mineral. 79, 9-14. 